\title{
Effect on Patient Safety of Brief Interventions Performed by Pharmacists via Drug Profile Books: VISualization of Treatment Assist by pharmacists (VISTA) Project in Japan
}

\author{
Masaki Shoji*, Mitsuko Onda, Yukio Arakawa \\ Clinical Laboratory of Practical Pharmacy, Osaka University of Pharmaceutical Sciences, Osaka, Japan \\ Email: "shoji@gly.oups.ac.jp
}

Received 6 April 2016; accepted 24 April 2016; published 27 April 2016

Copyright (C) 2016 by authors and Scientific Research Publishing Inc.

This work is licensed under the Creative Commons Attribution International License (CC BY). http://creativecommons.org/licenses/by/4.0/

cC) (i) Open Access

\begin{abstract}
In Japanese pharmacies, Drug Profile Books (DPBs), which are a type of Personal Health Record (PHR), are incorporated in order to prevent duplicate medication and drug interactions in outpatients (patients) through the uniform management of drug administration information. In this study, we tried to clarify the effect on patient safety of brief interventions via DPBs by pharmacists. The study design was a randomized controlled trial on pharmacies as clusters. 65 pharmacies agreed to participate in the study (intervention group (IG): 33; control group (CG): 32). The primary outcomes were: rate of inquiry occurrence, rate of prescription change, and rates of duplicate medications \& drug interactions. 56 pharmacies (IG: 29; CG: 27) completed the study. There was a higher tendency for prescription changes in the IG compared to the CG (IG: 0.03\%; CG: $0.02 \% ; P=0.08$ ). In addition, the rate of duplicate medications \& drug interactions accounting for the inquiries was significantly higher in the IG than in the CG (IG: 89.2\%; CG: 71.9\%; $P=0.01$ ). This implied that brief interventions by pharmacists using DPBs had an effect in raising patient safety.
\end{abstract}

\section{Keywords}

Drug Profile Book, Pharmaceutical Care, Community Pharmacy, Patient Safety, Brief Intervention, Communication, Personal Health Record

\footnotetext{
${ }^{*}$ Corresponding author.
}

How to cite this paper: Shoji M., Onda M. and Arakawa Y. (2016) Effect on Patient Safety of Brief Interventions Performed by Pharmacists via Drug Profile Books: VISualization of Treatment Assist by pharmacists (VISTA) Project in Japan. Pharmacology \& Pharmacy, 7, 176-183. http://dx.doi.org/10.4236/pp.2016.74023 


\section{Introduction}

Because the avoidance of adverse drug reactions is directly tied to patient safety, it is one of the duties at the heart of pharmaceutical care provided by pharmacists. Because patients in Japan can freely select medical institutions, many patients are examined by multiple medical institutions, and receive medications from multiple pharmacies. Therefore, unified management of their medication information by patients and the presentation of this to medical institutions, are necessary in order to ensure patient safety with regard to drug therapies [1]. Thus the Drug Profile Book (DPB) was developed by pharmacists as a Personal Health Record (PHR). The use of the DPB is a Japan-specific medical medium that has been in place since 1983. DPBs are mainly A6-sized handbooks, and very easy to use for the elderly as well. In addition, they can be used not only during examinations but also when purchasing OTC (over-the-counter) medications and supplements [2] [3], and for patient education at discharge [4]. The main purpose for using a DPB in routine medical care is to prevent duplicate medications and drug interactions before they happen and to avoid adverse drug reactions. With each patient's agreement, the pharmacist issues him or her with a DPB in which information is accumulated about the drugs the patient has been prescribed by affixing "the sticker" in the book, mainly carrying details about prescriptions dispensed by pharmacies [5]. When receiving treatment by a medical institution, the patient brings the DPB and presents it to the physician and pharmacist, and the physician can take action at that time based on the DPB information. In addition, pharmacists can provide proper dispensing and administration instructions based on the DPB information. However, there are often cases where the patient does not bring the DPB to the medical institution, the patient does not show the DPB to the treatment provider, or the patient has multiple DPBs and so on, and uniform management of drug administration information is still insufficient [6] [7]. There are reports that whether PHRs can be effectively used is affected by the understanding of the importance by the patient him/herself regarding PHR [8]. However, there are few reports dedicated solely to DPB. Therefore, the authors performed a cross-sectional study of patients undergoing treatment for hypertension. As a result, it became clear that the patients' rate of bringing the DPB to their pharmacy (rate of bringing the DPB) was correlated to the patients' recognition of the role and sense of utility [9]. In addition, patients with experience of having been questioned by a pharmacist while looking at the DPB are known to have a higher rate of bringing the DPB, recognition of the role, sense of utility, and experience of showing the DPB to the physician [5]. However, because these empirical studies had a cross-sectional design, it was difficult to verify the effect of communication via DPBs.

\section{Objective}

To clarify the effect on patient safety of brief interventions via DPBs by pharmacists.

\section{Methods}

\subsection{Study Design}

This study was an open label randomized controlled trial on pharmacies as clusters. The pharmacies participating in the study, after having registered to participate, were stratified, per company, by the number of pharmacists working per pharmacy, and were randomly assigned to either an intervention group (IG) or a control group (CG) by random numbers generated in Microsoft Excel 2007.

\subsection{Settings}

Participants in this study were community pharmacists and outpatients.

\subsection{Study Period and Locations}

We sought the cooperation of two dispensing pharmacy chains deployed mainly in Kansai and obtained agreements to participate in the study from 65 pharmacies in Osaka, Hyogo, Nara, Kyoto, Okayama, Toyama, Ishikawa, Aichi, Mie, Chiba, Kanagawa, and Tokyo. The intervention period was 3 months from 24 August 2015.

\subsection{Intervention Group}

The pharmacists of the IG were asked to follow instructions carefully regarding the following 2 points with all 
patients who came to the pharmacy with a DPB during the study period.

1) Checking the DPB together with the patient, and having discussions based on the information in the DPB

2) Performing entry in the DPB

With regard to: 1) they were requested to make comments on concomitant medication and check OTC medications each time during usual administration instructions; 2) they were requested to proactively enter such information as the quantity of drugs remaining, OTC medications, and supplements during administration. In addition, in the IG we requested that there be a meeting each morning, and to be sure to share information between the staff on cases such as duplicate administrations and interactions, etc., prevented by the intervention. Even in cases where there were no cases worthy of sharing, we were careful to stress that the fact of there being "no cases worth sharing" be shared. In addition, for purposes of informing the staff we displayed posters in the dispensing room that a study is being implemented.

\subsection{Control Group}

In the CG we requested that administration instructions be performed as before, and did not request periodic information sharing regarding cases or the hanging of informational posters.

\subsection{Verification of Primary Outcomes}

During the study period, the pharmacists of both the IG and CG were distributed a questionnaire for the handbooks, one handbook per pharmacist, and requested to always carry these within their white coats while working. Then they were requested, when cases occurred, to record the content thereof in the questionnaire. The main items of the questionnaire were: 1) the content of the case; 2) whether the pharmacist did inquiry to the physician; and 3) the result of the inquiry. The following items were set as the primary outcomes.

1) Rate of inquiry occurrence

The rate of inquiries that occurred based on the DPB information relative to the number of prescriptions received to during the study period.

2) Rate of prescription changes

Proportion of the number of inquiries where prescription changes occurred relative to the number of prescriptions received to during the study period.

3) Proportion of duplicate medications and drug interactions

Proportion of the number of inquiries related to duplicate medications and drug interactions relative to the number of inquiries.

\subsection{Verification of Secondary Outcomes}

Patients were recruited for 1 week at each pharmacy, the gist of the study was explained with the informed consent form, and having obtained consent to participate, the questionnaire was distributed at the start of the study as well as at the end of the intervention period (after 3 months) and a reply requested. The patient inclusion (exclusion) criteria are shown below.

1) Patient inclusion criteria

a) Persons having their own DPB

b) Persons who can be expected to come to the pharmacy continuously for 3 months

c) Persons thought capable of responding to the questionnaire

d) Persons with a prescription term within 1 month

\section{2) Patient exclusion criteria}

a) Persons not having their own DPB

b) Persons who cannot be expected to come to the pharmacy continuously for 3 months

c) Persons suffering from conditions such as dementia that make it difficult to answer

d) Persons who have representatives other than children or spouses come to pick up the medications

The following items were set as the secondary outcomes, in addition to the patient attributes:

1) Rate of bringing the DPB

We requested, with regard to the frequency of the patient bringing the DPB to the pharmacy, that the patient self-report their best estimate using a number from $0 \%-100 \%$. 
2) The experience of showing the DPB to the physician

We requested, with regard to the question "Do you show the DPB to your physician?", that the response be on a 3-step scale of "I always show it", "I sometimes show it", and "I don't show it". The proportion responding other than "I don't show it" was treated as "the experience of showing the DPB to the physician".

3) Proper use of the DPB

We requested a response on a 3-step scale regarding the following 4 items about "I know and am practicing it", "I know but am not practicing it", and "did not know": a) do not use multiple DPBs at the same time. Manage with a single DPB; b) a sticker must be affixed each time even if the drug content is the same; c) OTC drugs and health foods being taken may also be entered; d) can be used as a health handbook by entering test values, etc. We then performed scoring where "I know and am practicing it" $=1$, and all others $=0$.

4) Recognition of the role

We requested, with regard to the question "Do you understand the role of the DPB?" that the response be on a 5-step scale of "I think so - I don't think so". We then performed scoring where "I think so" = 5 "I don't think so" $=1$.

5) Sense of utility

We requested, with regard to the question "Is the DPB helpful for you?" that the response be on a 5-step scale of "I think so - I don't think so", respectively. We then performed scoring where "I think so" $=5 \sim$ "I don't think so" $=1$.

\subsection{Statistical Analysis}

\subsubsection{Analysis of Primary Outcomes}

We compared the occurrence rate of inquiries, the rate of prescription changes, and the rates of duplicate medication and drug interaction in the IG and CG using Pearson's $\chi^{2}$ test.

\subsubsection{Analysis of Secondary Outcomes}

We compared the patient attributes (age, sex, number of administered medications, number of conditions) rate of bringing the DPB, experience of showing the DPB to the physician, proper use of the DPB, recognition of the role, and sense of utility using the Mann Whitney U test and Pearson's $\chi^{2}$ test. Furthermore, PASW Statistics 18 was used for all statistical analyses, and the significance level was set at $5 \%$.

\subsection{Ethical Considerations}

This study was performed after having obtained the approval (Approval number: 0023) of the Osaka University of Pharmaceutical Sciences Institutional Review Board.

\section{Results}

\subsection{Pharmacy Attributes}

The 65 pharmacies were randomly assigned to the IG $(n=33)$ and the $C G(n=32)$. During the study period, 4 pharmacies dropped out in the IG, and 5 in the CG, and 29 pharmacies completed the study in the IG and 27 in the CG. Table 1 shows the pharmacy attributes at baseline. No significant differences were seen between the IG and the CG.

Table 1. Baseline characteristics of cluster.

\begin{tabular}{ccc}
\multicolumn{1}{c}{ Variables } & IG & CG \\
\hline Cluster Number of pharmacists & $\mathbf{n}=\mathbf{2 9}$ & $\mathbf{n}=\mathbf{2 7}$ \\
$1 \sim 3$ & 4 & 4 \\
$4 \sim 5$ & 8 & 7 \\
$6 \sim 7$ & 8 & 8 \\
$8 \sim$ & 9 & 8 \\
\hline
\end{tabular}




\subsection{Patient Attributes}

In the IG 345 patients, and in the CG 229 patients, agreed to reply to the questionnaire. During the study period, 24 patients dropped out in the IG, and 65 in the CG, and 321 patients completed the study in the IG and 164 in the CG (Figure 1). Table 2 shows the patient attributes at baseline. No significant differences were seen between the IG and the CG.

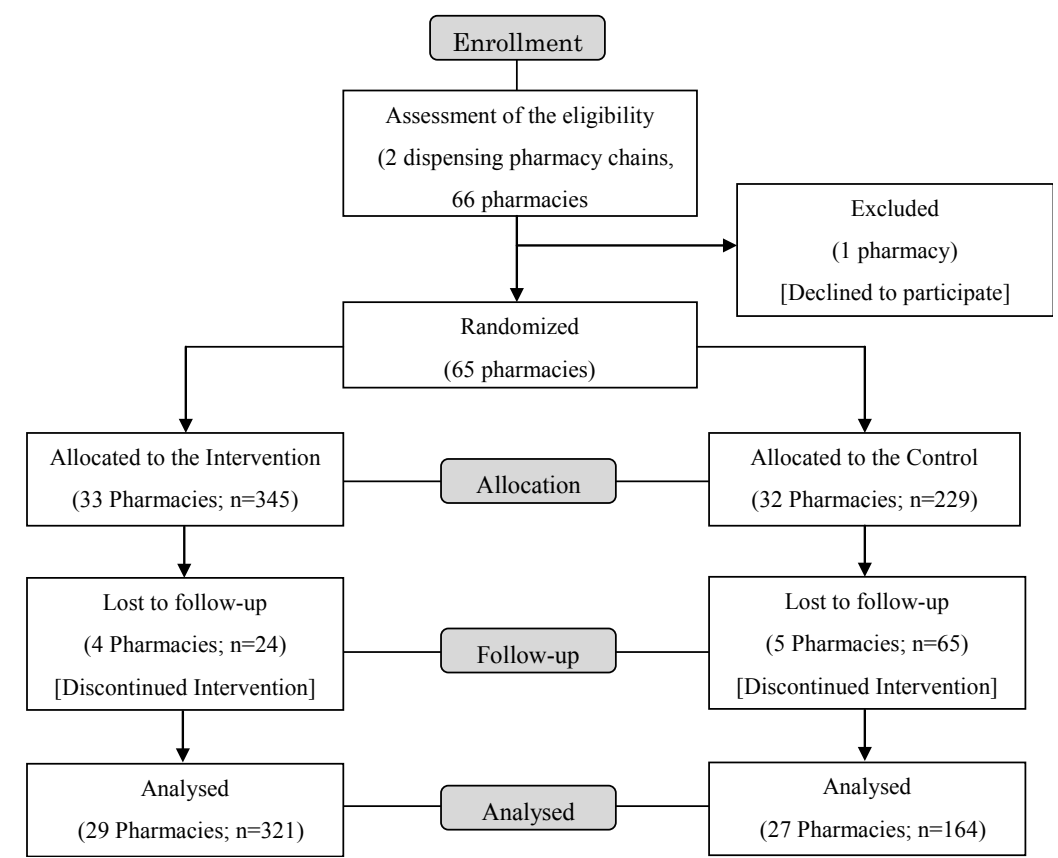

Figure 1. Flow of the participants through VISTA project.

Table 2. Baseline characteristics of patients.

\begin{tabular}{lcc}
\hline Variables & IG $(\mathbf{n}=\mathbf{3 2 1})$ & CG (n= 164) \\
\hline Age [mean (SD)] & $66.8(15.4)$ & $65.7(16.3)$ \\
Gender (Male) [n (\%)] & $119(38.1)$ & $56(34.4)$ \\
Number of taken drug [mean (SD)] & $6.2(3.8)$ & $5.6(3.8)$ \\
Number of complicating disease [mean (SD)] & $1.8(1.0)$ & $1.9(1.0)$ \\
Diseases (multiple choice) [n (\%)] & & $59(36.0)$ \\
Hypertension & $103(32.1)$ & $34(20.7)$ \\
Hyperlipidemia & $68(21.2)$ & $22(13.4)$ \\
Diabetes & $39(12.1)$ & $17(10.4)$ \\
Cardiac disorder & $26(8.1)$ & $6(3.7)$ \\
Cerebrovascular disorder & $11(3.4)$ & $23(14.0)$ \\
Digestive disorder & $37(11.5)$ & $13(7.9)$ \\
Chronic pain & $42(13.1)$ & $20(12.2)$ \\
Osteoporosis & $21(6.5)$ & $16(9.8)$ \\
Sleep disorder & $23(7.2)$ & $11(6.7)$ \\
Psychiatric disorder & $19(5.9)$ & $10(6.1)$ \\
Respiratory disorder & $22(6.9)$ & $3(1.8)$ \\
Urinary organ disorder & $10(3.1)$ & $3(1.8)$ \\
Cutaneous disorder & $12(3.7)$ & $1(0.6)$ \\
Renal disorder & $8(2.5)$ & $23(14.0)$ \\
Others & $67(20.9)$ & \\
\hline
\end{tabular}




\subsection{Primary Outcomes}

The number of all the prescriptions received during the study period was 180,398 in the IG and 182,757 in the CG. Although no significant difference was seen in the occurrence rate of inquiries [IG: 0.04\% (74); CG: $0.03 \%$ (57); $\mathrm{P}=0.13]$, a tendency for more prescription changes was seen in the IG [IG: $0.03 \%$ (57); CG: $0.02 \%(40)$; $\mathrm{P}=0.08]$. In addition, the rate of duplicate medications \& drug interactions was significantly higher in the IG than in the CG [IG: $89.2 \%$ (66); CG: 71.9\% (41); P = 0.01] (Table 3).

\section{Table 3. Primary outcomes.}

\begin{tabular}{|c|c|c|c|}
\hline & IG & CG & $\mathbf{P}$ \\
\hline Total number of prescription scrips during the study period & 180,398 & 182,757 & \\
\hline Occurrence Rate of Inquiries [\% (Cases $)^{\mathrm{a}}$ ] & $0.04(74)$ & $0.03(57)$ & 0.13 \\
\hline Rate of Prescription Changes [\% (Cases $\left.)^{\mathrm{b})}\right]$ & $0.03(57)$ & $0.02(40)$ & 0.08 \\
\hline Duplicate medications and Drug interactions $\left[\%(\mathrm{Cases})^{\mathrm{c}}{ }^{\mathrm{j}}\right]$ & $89.2(66)$ & $71.9(41)$ & 0.01 \\
\hline Duplicate medication & $82.4(61)$ & $66.7(38)$ & 0.04 \\
\hline Drug interaction & $6.8(5)$ & $5.2(3)$ & 0.72 \\
\hline Others $^{\text {d) }}$ & $10.8(8)$ & $28.1(16)$ & 0.01 \\
\hline
\end{tabular}

Pearson's $\chi^{2}$ test; a) Proportion of the number of inquiries that occurred based on the DPB information relative to the number of prescriptions received during the study period; b) Proportion of the number of prescription changes that occurred relative to the number of prescription scrips responded to during the study period; c) Proportion of the number of questions related to duplicate medications and drug interactions relative to the number of inquiries; d) "Others" included history of drug allergies, history of adverse reactions, aimless administration, and others.

\subsection{Secondary Outcomes}

The results of having compared the secondary outcomes between the IG and the CG at baseline and after study completion are shown in Table 4.

Table 4. Secondary outcomes.

\begin{tabular}{|c|c|c|c|c|c|c|}
\hline & \multicolumn{2}{|c|}{ Baseline } & \multicolumn{4}{|c|}{ After 12 week } \\
\hline & $\begin{array}{c}\text { IG } \\
(n=321)\end{array}$ & $\begin{array}{c}\text { CG } \\
(n=164)\end{array}$ & $\mathbf{P}$ & $\begin{array}{c}\text { IG } \\
(n=321)\end{array}$ & $\begin{array}{c}C G \\
(n=164)\end{array}$ & $\mathbf{P}$ \\
\hline Rate of bringing the DPB $[(\%) \text { mean }(\mathrm{SD})]^{\mathrm{a})}$ & $89.1(19.1)$ & $87.6(23.8)$ & $0.55^{1)}$ & $92.3(16.1)$ & $88.5(20.0)$ & $0.03^{1)}$ \\
\hline The experience of showing the DPB to the physician $(\%)^{\mathrm{b})}$ & 37.2 & 33.8 & $0.46^{2)}$ & 47.2 & 40.8 & $0.19^{2}$ \\
\hline Proper use of the DPB [mean (SD) $]^{\mathrm{c})}$ & $2.0(1.0)$ & $2.0(1.0)$ & $0.99^{1)}$ & $2.3(1.0)$ & $2.0(1.1)$ & $0.01^{1)}$ \\
\hline Recognition of the role $[\text { mean }(\mathrm{SD})]^{\mathrm{d})}$ & $4.5(1.0)$ & $4.4(1.1)$ & $0.76^{1)}$ & $4.6(0.9)$ & $4.5(0.9)$ & $0.01^{1)}$ \\
\hline Sense of utility [mean (SD) $]^{\mathrm{e})}$ & $4.5(1.0)$ & $4.4(1.1)$ & $0.95^{1)}$ & $4.6(0.9)$ & $4.4(1.0)$ & $0.17^{1}$ \\
\hline
\end{tabular}

1) Mann-Whitney's U test; 2) Pearson's $\chi^{2}$ test; a) The patient entered a self-evaluation as an actual number from $0 \% \sim 100 \%$; b) Selection rate for the selections "I always show it" and "I sometimes show it" to the question "Do you show the DPB to your physician?"; c) Total score regarding 4 question items about use: 1 . Do not use concomitantly multiple DPBs at the same time. Manage with a single DPB. 2. A sticker must be affixed each time even if the drug content is the same. 3. OTC drugs and health foods being taken are good also for being entered. 4 . Can be used as a health handbook by entering test values; d) Score of the responses on a 5-step scale of "I think so (=5)" - I don't think so (=1)" with regard to the question "Do you understand the role of the DPB?"; e) Score of the responses on a 5-step scale of "I think so (=5)" - I don't think so (=1)" with regard to the question "Is the DPB helpful for you?"

Although no difference was seen between the IG and the CG in any of the items at baseline, at the completion of the study the rate of bringing the DPB (IG: $92.3 \%$; CG88.5\%; P = 0.03), proper use of the DPB (IG: 2.3; CG: $2.0 ; \mathrm{P}=0.01$ ), and recognition of the role (IG: $4.6 ; \mathrm{CG}: 4.5 ; \mathrm{P}=0.01$ ) had significantly risen in the IG compared to the $\mathrm{CG}$.

\section{Discussion}

This study revealed that brief interventions by pharmacists improved patient safety. First, a tendency for more prescription changes was seen in the IG compared to the CG. This implied that inquiries for content that affected the prescription behavior of physicians had increased. For this reason, a significant increase was evidenced in 
the rates of duplicate medication and drug interaction, and particularly those caused by duplicate medication, in the IG compared to the CG. This is thought to be brought about by the opportunities for pharmacists to directly obtain information related to concomitant medications and the use of OTC medications and supplements, etc., from patients, promoting unified management of drug administration information. The results showed that enhancement of pharmacist communication with patients via DPBs prevented duplicate medications and drug interactions, which was the main purpose for using DPBs, and showed a contribution in raising the accuracy of adverse drug reaction avoidance.

Second, the rate of bringing the DPB was significantly higher at the end of the study in the IG compared to the CG. The reason was thought be that the pharmacists of the IG promoted regular bringing of the DPB when patients brought a DPB, and likewise educated patients when they did not bring a DPB. This might have occurred due to the periodic information sharing between the staff members regarding inquiry cases in the IG helping to motivate pharmacists to educate patients.

Third, the proper use of the DPB and the recognition of the role at the end of the study were significantly higher in the IG than in the CG. The reason for this might be that patient awareness of the correct use of the DPB was enhanced by education by the pharmacist, consequently raising patients' understanding of the role of the DPB. The rate of bringing the DPB, proper use of the DPB, and recognition of the role are indispensable items for the unified management of drug administration information at pharmacies. Therefore, the elevation of these secondary outcomes was thought to be tied to the difference in primary outcomes.

On the other hand, no difference was seen in the occurrence rate of inquiries between the IG and the CG. This implies that inquiries are a mandatory duty of pharmacists, and that the enhancement of communication via DPBs is not correlated to the number of actual inquiries. In addition, no difference was seen in the sense of utility between the IG and the CG. Proposed reasons therefore, that there were no differences in the patient attributes at baseline and after study completion between the two groups. The authors have already shown the correlations between differences in sense of utility with patient attributes (age, sex, and number of medications administered) [8].

With regard to the experience of showing the DPB to the physician, even though no statistically significant differences were seen between the IG and CG, in the IG this had risen $10.0 \%$ from baseline to completion, a 3.0 point difference compared to $7.0 \%$ in the CG. Because it has been reported that $92.3 \%$ of Japanese physicians have experience of having avoided adverse drug reactions by the use of DPBs [9], it is thought that education by pharmacists to show DPBs to physicians is significant in raising patient safety.

Finally, this study has some limitations. First, because there is no literature pertaining to interventional studies using DPBs, it was not possible to accurately calculate a sample size suitable for verification of the secondary outcomes. Currently, we identified the patient inclusion (exclusion) criteria, and requested recruitment of 30 persons as the upper limit, in consideration of the feasibility of study implementation at pharmacies. As a result, because there was no great bias in the mean values of the number of patients recruited by the pharmacies, with 10.5 persons in the IG and 7.2 in the CG, this is not thought to have had a large effect on the results.

Second, the rate of bringing the DPB in both the IG and the CG was higher compared to the values of previous studies [3] [10], and it was possible that this did not reflect the true state of the population. This might be due to the initially high level of awareness of the patients participating in this study regarding the bringing of DPBs. Third, the number of patients in the CG was smaller than that of the IG. Here, the reason might have been that in the IG the pharmacists enhanced the communication via DPBs, and thereby maintained the patients' motivation to participate in the study. However, additional recruitment for the CG was not performed in order to avoid creating bias in patient attributes and numbers of clusters.

Although there were some limitations, this study was the first in Japan where the effect of brief interventions using DPBs by pharmacists was verified, and is thought to be significant in that it specifically presented a method practicable in daily work duties to enhance the unified management of drug administration information by pharmacists. From these findings, authors think that taking communication via medical information device like DPBs is meaningful for patient safety, and it also develop the relationship between pharmacists and patients.

\section{Acknowledgements}

The authors would like to acknowledge the participation of the following pharmacies and pharmacists in making this study possible: Saera Pharmacy Co., Ltd., Japan Pharmacy Co., Ltd. We'd like to thank Mr. Kentaro Iwade 
and Mr. Makoto Sakino of Saera Pharmacy, Mr. Maeda Takahiro of Japan Pharmacy, and Mr. Nobuaki Hayashi of Hayashi Print, as well as the pharmacists of the cooperating pharmacies, to all our deepest thanks.

\section{Conflicts of Interest}

There were no issues associated with conflicts of interest in this study.

\section{References}

[1] Iijima, H., Ishino, Y., Ando, H. and Mogi, H. (2005) Use of Patient Information to Enhance Drug Information Provided by Pharmacies. Japanese Journal of Pharmaceutical Health Care and Sciences, 31, 223-227. http://dx.doi.org/10.5649/jiphcs.31.223

[2] Hosaka, E., Takayanagi, R., Suzuki, A., Orii, T., Seino, T., Shimizu, H., Yamamura, Y., Nakamura, K., Kotaki, H., Sawada, Y. and Iga, T. (1997) Evaluation of the Efficacy of a Prescription Information System for the Rational Use of Medications - Patient Consultations Using a "Drug History Handbook". Journal of the Nippon Hospital Pharmacists Association, 23, 342-347. http://dx.doi.org/10.5649/jiphcs1975.23.342

[3] Yamamura, K., Masuda, M., Kuramochi, K., Ishizuka, T., Someya, T., Suganuma, Y. and Motohashi, H. (2003) Investigation of the "Medication Notebook" about Its Utilization in Patients and Its Evaluation by Doctors in Iwai City. YAKUGAKU ZASSHI, 123, 151-155. http://dx.doi.org/10.1248/yakushi.123.151

[4] Tachi, T., Asano, S., Goto, C., Yoshida, T., Usui, K., Kano, M., Yokoi, T., Tanaka, K., Yasuda, M., Mizui, T. and Teramachi, H. (2015) Effect of Group Instructions for Improving Awareness about Medication-Notebook Use on the Purchase of Over-the-Counter Drugs and Dietary Supplements. Japanese Journal of Pharmaceutical Health Care and Sciences, 41, 113-120. http://dx.doi.org/10.5649/jjphcs.41.113

[5] Shoji, M., Iwade, K., Keiko, F., Hirota, M., Kanou, A., Moriya, M., Ishii, M., Shimoji, S., Onda, M. and Arakawa, Y. How Patient-Pharmacist Communication Using the Drug Profile Book Relates to Patient's Behavior Regarding Its Use. YAKUGAKU ZASSHI. (In Press)

[6] Ojima, F., Takeda, N., Takeda, M., Sakurai, K., Handa, M., Aihara, Y., Mineta, J., Nitta, Y., Ito, J., Okazaki, C., Nakagawa, Y. and Watanabe, Y. (2007) Investigation of the Usefulness of Medication Notebooks No. 4: Questionnaire on Awareness of Medication Notebooks and Their Usage among Doctors in Yamagata City. Japanese Journal of Pharmaceutical Health Care and Sciences, 33, 54-59. http://dx.doi.org/10.5649/ijphcs.33.54

[7] Ueki, T., Miyano, Y., Sakamoto, Y., Nagai, K., Hashiguchi, Y., Nakashima, M. and Nakashima, M. (2015) A Comparative Study between Emergent and Elective Admission: Identifying Medicines Brought to Hospital by Inpatients. Japanese Journal of Drug Informatics, 17, 39-44. http://doi.org/10.11256/jjdi.17.39

[8] Tang, P.C., Ash, J.S., Bates, D.W., Overhage, J.M. and Sands, D.Z. (2006) Personal Health Records: Definitions, Benefits, and Strategies for Overcoming Barriers to Adoption. Journal of the American Medical Informatics Association, 13, 121-126. http://dx.doi.org/10.1197/jamia.M2025

[9] Shoji, M., Onda, M., Iwade, K. and Arakawa, Y. (2015) Study on the Factors Affecting the Frequency of Bringing the Drug Profile Book and of Using the Stickers in Patients Taking Antihypertensive. Japanese Journal of Pharmaceutical Health Care and Sciences, 41, 139-146. http://dx.doi.org/10.5649/jjphcs.41.139

[10] Ide, K., Ikumi, Y., Kiuchi, A., Sone, J., Kojima, M. and Yamada, H. (2015) Submission Rates of Medication Notebook in Insurance Pharmacies. Japanese Journal of Drug Informatics, 16, 201-205. http://doi.org/10.11256/jjdi.16.201 